Copyright by the Acoustical Society of America. Reddy, J. N., \& Khdeir, A. A. (1989). Dynamic response of cross-ply laminated shallow shells according to a refined shear deformation theory. Journal of the Acoustical Society of America, 85(6), 2423-2431. doi: 10.1121/1.397791

\title{
Dynamic response of cross-ply laminated shallow shells according to a refined shear deformation theory
}

\author{
J. N. Reddy and A. A. Khdeir \\ Department of Engineering Science and Mechanics, Virginia Polytechnic Institute and State University, \\ Blacksburg, Virginia 24061
}

(Received 13 May 1988; accepted for publication 10 January 1989)

The dynamic response of cross-ply laminated shallow shells is investigated using the thirdorder shear deformation shell theory of Reddy [J. Appl. Mech. 41, 47 (1984) ]. The theory accounts for cubic variation of the in-plane displacements through the thickness and does not require shear correction coefficients. The state-space approach is used to develop the analytical solutions of simply supported, cross-ply shells using the classical, first-order, and higher-order theories. The use of the separation of variables technique for the higher-order theory is also presented. Numerical results of the higher-order theory for center deflection and normal stresses of spherical shells under various loadings are compared with those obtained using the classical and first-order [or Sanders, Q. Appl. Math. 21, 21-36 (1963)] shell theories.

PACS numbers: 43.40.At, 43.40.Ey

\section{INTRODUCTION}

Analytical description of laminated composite shells is often based on the classical laminate shell theory, which is an extension of the Love-Kirchhoff (or the first-approximation) shell theory to composite shells. In the classical shell theory, the transverse strains are neglected under the assumption that the straight lines normal to the middle surface are rigid (i.e., do not deform). The neglect of transverse shear strains in composite laminates could lead to underestimation of deflections and overestimation of natural frequencies and critical buckling loads because of the low transverse shear modulus compared to the in-plane Young's moduli. ${ }^{1}$

Refined theories are those in which the transverse strains are accounted for. Often these theories are developed from an assumed displacement field. The expansion of displacements as a linear combination of various powers of the thickness coordinate and undetermined functions of shell surface coordinates was suggested by Basset. ${ }^{2}$ Hildebrand $e t$ $a l .{ }^{3}$ used a linear expansion of the displacements to develop a shear deformation shell theory (see also Sanders ${ }^{4}$ ). Whitney and Sun $^{5,6}$ developed a refined shell theory in which the displacements in the surface of the shell are expanded as linear functions of the thickness coordinate and the transverse displacement is expanded as a quadratic function of the thickness coordinate. Reddy ${ }^{7}$ and Reddy and $\mathrm{Liu}^{8}$ presented a third-order theory in which the surface displacements are expanded up to the cubic term in thickness coordinate, while the transverse deflection is assumed to be constant through the thickness. The nine undetermined functions are reduced to five by imposing stress-free boundary conditions on the transverse shear stresses on the bounding surfaces of the shell. The theory does not require any shear correction coefficients, which are used in the first-order shell theories.

The objective of the present study is to investigate the transient behavior of laminated, cross-ply, composite shell panels using the third-order Shell theory ${ }^{8}$ and to compare the results with those obtained using the classical and firstorder shell theories. Analytical solutions of the theories are obtained using the method of state-space technique, and separation of variables approach ${ }^{9-11}$ for higher-order theory.

\section{HIGHER-ORDER SHEAR DEFORMATION THEORY (HSDT)}

The third-order theory used in the present study is based on the following displacement field (see Reddy and $\mathrm{Liu}^{8}$ ):

$$
\begin{aligned}
& \bar{u}=\left(1+\frac{\zeta}{R_{1}}\right) u+\zeta \phi_{1}+\zeta^{3} \frac{4}{3 h^{2}}\left(-\phi_{1}-\frac{1}{\alpha_{1}} \frac{\partial w}{\partial \xi_{1}}\right), \\
& \bar{v}=\left(1+\frac{\zeta}{R_{2}}\right) v+\zeta \phi_{2}+\zeta^{3} \frac{4}{3 h^{2}}\left(-\phi_{2}-\frac{1}{\alpha_{2}} \frac{\partial w}{\partial \xi_{2}}\right),
\end{aligned}
$$

$$
\bar{w}=w,
$$

where $(\bar{u}, \bar{v}, \bar{w})$ are the displacements along the orthogonal curvilinear coordinates such that the $\xi_{1}$ and $\xi_{2}$ curves are lines of principal curvature on the midsurface $\zeta=0$, and $\zeta$ curves are straight lines perpendicular to the surface $\zeta=0$, $(u, v, w)$ are the displacements of a point on the middle surface, and $\phi_{1}$ and $\phi_{2}$ are the rotations at $\xi=0$ of normals to the midsurface with respect to the $\xi_{2}$ and $\xi_{1}$ axes, respectively. The parameters $R_{1}$ and $R_{2}$ denote the values of the principal radii of curvature of the middle surface, and $\alpha_{1}$ and $\alpha_{2}$ are the surface matrices defined in Refs. 1 and 8. All displacement components $\left(u, v, w, \phi_{1}, \phi_{2}\right)$ are functions of $\left(\xi_{1}, \xi_{2}\right)$ and time $t$.

Substituting Eq. (1) into the strain-displacement relations of a shell referred to an orthogonal curvilinear coordinate system, we obtain

$$
\begin{aligned}
& \epsilon_{1}=\epsilon_{1}^{0}+\zeta\left(\kappa_{1}^{0}+\zeta^{2} \kappa_{1}^{2}\right), \\
& \epsilon_{2}=\epsilon_{2}^{0}+\zeta\left(\kappa_{2}^{0}+\zeta^{2} \kappa_{2}^{2}\right), \\
& \epsilon_{4}=\epsilon_{4}^{0}+\zeta^{2} \kappa_{4}^{1}, \\
& \epsilon_{5}=\epsilon_{5}^{0}+\zeta^{2} \kappa_{5}^{1}, \\
& \epsilon_{6}=\epsilon_{6}^{0}+\zeta\left(\kappa_{6}^{0}+\zeta^{2} \kappa_{6}^{2}\right),
\end{aligned}
$$

where 


$$
\begin{aligned}
& \epsilon_{1}^{0}=\frac{\partial u}{\partial x_{1}}+\frac{w}{R_{1}}, \quad \kappa_{1}^{0}=\frac{\partial \phi_{1}}{\partial x_{1}}, \quad \kappa_{1}^{2}=-c_{2}\left(\frac{\partial \phi_{1}}{\partial x_{1}}+\frac{\partial^{2} w}{\partial x_{1}^{2}}\right) \\
& \epsilon_{2}^{0}=\frac{\partial v}{\partial x_{2}}+\frac{w}{R_{2}}, \quad \kappa_{2}^{0}=\frac{\partial \phi_{2}}{\partial x_{2}}, \quad \kappa_{2}^{2}=-c_{2}\left(\frac{\partial \phi_{2}}{\partial x_{2}}+\frac{\partial^{2} w}{\partial x_{2}^{2}}\right) \\
& \epsilon_{4}^{0}=\phi_{2}+\frac{\partial w}{\partial x_{2}}, \quad \kappa_{4}^{1}=-c_{1}\left(\phi_{2}+\frac{\partial w}{\partial x_{2}}\right) \\
& \epsilon_{5}^{0}=\phi_{1}+\frac{\partial w}{\partial x_{1}}, \quad \kappa_{5}^{1}=-c_{1}\left(\phi_{1}+\frac{\partial w}{\partial x_{1}}\right) \\
& \epsilon_{6}^{0}=\frac{\partial v}{\partial x_{1}}+\frac{\partial u}{\partial x_{2}}, \quad \kappa_{6}^{0}=\frac{\partial \phi_{2}}{\partial x_{1}}+\frac{\partial \phi_{1}}{\partial x_{2}} \\
& \kappa_{6}^{2}=-c_{2}\left(\frac{\partial \phi_{2}}{\partial x_{1}}+\frac{\partial \phi_{1}}{\partial x_{2}}+2 \frac{\partial^{2} w}{\partial x_{1} \partial x_{2}}\right)
\end{aligned}
$$

Here, $x_{i}$ denote the Cartesian coordinates $\left(d x_{i}=\alpha_{i} d \xi_{i}\right.$, $i=1,2)$, and $c_{1}=4 / h^{2}$ and $c_{2}=c_{1} / 3$.

The stress-strain relations for the $k$ th lamina are given by

$$
\begin{aligned}
\left(\begin{array}{l}
\sigma_{1} \\
\sigma_{2} \\
\sigma_{6} \\
\sigma_{4} \\
\sigma_{5}
\end{array}\right)_{(k)} \\
=\left(\begin{array}{lllll}
Q_{11}^{(k)} & Q_{12}^{(k)} & Q_{16}^{(k)} & 0 & 0 \\
& Q_{22}^{(k)} & Q_{26}^{(k)} & 0 & 0 \\
& & Q_{66}^{(k)} & 0 & 0 \\
\text { symm. } & & & Q_{44}^{(k)} & 0 \\
& & & & Q_{55}^{(k)}
\end{array}\right)\left(\begin{array}{l}
\epsilon_{1} \\
\epsilon_{2} \\
\epsilon_{6} \\
\epsilon_{4} \\
\epsilon_{5}
\end{array}\right),
\end{aligned}
$$

where $Q_{i j}^{(k)}$ are the material constants of the $k$ th lamina in the laminate coordinate system.

Using Hamilton's principle, the equations of motion appropriate for the displacement field (1) and the constitutive equations (4) are derived in Ref. 8 as

$$
\begin{aligned}
& \frac{\partial N_{1}}{\partial x_{1}}+\frac{\partial N_{6}}{\partial x_{2}}+g_{1}=\bar{I}_{1} \ddot{u}+\bar{I}_{2} \ddot{\phi}_{1}-\bar{I}_{3} \frac{\partial \ddot{w}}{\partial x_{1}}, \\
& \frac{\partial N_{6}}{\partial x_{1}}+\frac{\partial N_{2}}{\partial x_{2}}+g_{2}=\bar{I}_{1}^{\prime} \ddot{v}+\bar{I}_{2}^{\prime} \ddot{\phi}_{2}-\bar{I}_{3}^{\prime} \frac{\partial \ddot{w}}{\partial x_{2}}, \\
& \frac{\partial Q_{1}}{\partial x_{1}}+\frac{\partial Q_{2}}{\partial x_{2}}-c_{1}\left(\frac{\partial K_{1}}{\partial x_{1}}+\frac{\partial K_{2}}{\partial x_{2}}\right) \\
& +c_{2}\left(\frac{\partial^{2} P_{1}}{\partial x_{1}^{2}}+\frac{\partial^{2} P_{2}}{\partial x_{2}^{2}}+2 \frac{\partial^{2} P_{6}}{\partial x_{1} \partial x_{2}}\right) \\
& -\frac{N_{1}}{R_{1}}-\frac{N_{2}}{R_{2}}+q \\
& =\bar{I}_{3} \frac{\partial \ddot{u}}{\partial x_{1}}+\bar{I}_{5} \frac{\partial \ddot{\phi}_{1}}{\partial x_{1}}+\bar{I}_{3}^{\prime} \frac{\partial \ddot{v}}{\partial x_{2}}+\bar{I}_{5}^{\prime} \frac{\partial \ddot{\phi}_{2}}{\partial x_{2}}+I_{1} \ddot{w} \\
& \quad-c_{2}^{2}\left(\frac{\partial^{2} \ddot{w}}{\partial x_{1}^{2}}+\frac{\partial^{2} \ddot{w}}{\partial x_{2}^{2}}\right),
\end{aligned}
$$

$$
\begin{aligned}
\frac{\partial M_{1}}{\partial x_{1}} & +\frac{\partial M_{6}}{\partial x_{2}}-Q_{1}+c_{1} K_{1}-c_{2}\left(\frac{\partial P_{1}}{\partial x_{1}}+\frac{\partial P_{6}}{\partial x_{2}}\right)+m_{1} \\
& =\bar{I}_{2} \ddot{u}+\bar{I}_{4} \ddot{\phi}_{1}-\bar{I}_{5} \frac{\partial \ddot{w}}{\partial x_{1}}
\end{aligned}
$$

$$
\begin{aligned}
\frac{\partial M_{6}}{\partial x_{1}} & +\frac{\partial M_{2}}{\partial x_{2}}-Q_{2}+c_{1} K_{2}-c_{2}\left(\frac{\partial P_{6}}{\partial x_{1}}+\frac{\partial P_{2}}{\partial x_{2}}\right)+m_{2} \\
= & \bar{I}_{2}^{\prime} \ddot{v}+\bar{I}_{4}^{\prime} \ddot{\phi}_{2}-\bar{I}_{5}^{\prime} \frac{\partial \ddot{w}}{\partial x_{2}},
\end{aligned}
$$

where superposed dots denote differentiation with respect to time, $\left(g_{1}, g_{2}\right)$ and $\left(m_{1}, m_{2}\right)$ are the body forces and moments, respectively, $q$ is the distributed transverse load, and $N_{i}, M_{i}$, etc., are the stress resultants

$$
\begin{aligned}
& \left(N_{i}, M_{i}, P_{i}\right)=\sum_{k=1}^{N} \int_{\zeta k-1}^{\zeta k} \sigma_{i}^{(k)}\left(1, \zeta, \zeta^{3}\right) d \zeta \quad(i=1,2,6) \\
& \left(Q_{1}, K_{1}\right)=\sum_{k=1}^{N} \int_{\zeta k-1}^{\zeta k} \sigma_{5}^{(k)}\left(1, \zeta^{2}\right) d \zeta \\
& \left(Q_{2}, K_{2}\right)=\sum_{k=1}^{N} \int_{\zeta k-1}^{\zeta k} \sigma_{4}^{(k)}\left(1, \zeta^{2}\right) d \zeta
\end{aligned}
$$

The inertias $I_{i}$ and $I_{i}^{\prime}(i=1,2,3,4,5)$ are defined by the equations

$$
\begin{aligned}
& \bar{I}_{1}=I_{1}+\left(2 / R_{1}\right) I_{2}, \\
& \bar{I}_{2}=I_{2}+\left(1 / R_{1}\right) I_{3}-c_{2} I_{4}-\left(c_{2} / R_{1}\right) I_{5}, \\
& \bar{I}_{3}=c_{2} I_{4}+\left(c_{2} / R_{1}\right) I_{5}, \\
& \bar{I}_{4}=I_{3}-2 c_{2} I_{5}+c_{2}^{2} I_{7}, \\
& \bar{I}_{5}=c_{2} I_{5}-c_{2}^{2} I_{7}, \\
& \left(I_{1}, I_{2}, I_{3}, I_{4}, I_{5}, I_{7}\right) \\
& \quad=\sum_{k=1}^{N} \int_{\zeta k-1}^{\zeta k} \rho^{(k)}\left(1, \zeta, \zeta^{2}, \zeta^{3}, \zeta^{4}, \zeta^{6}\right) d \zeta,
\end{aligned}
$$

and $\bar{I}_{i}^{\prime}$ are the same as $\bar{I}_{i}$ except that $R_{1}$ is replaced by $R_{2}$. The resultants are related to the total strains by

$$
\begin{aligned}
& N_{i}=A_{i j} \epsilon_{j}^{0}+B_{i j} \kappa_{j}^{0}+E_{i j} \kappa_{j}^{2}, \\
& M_{i}=B_{i j} \epsilon_{j}^{0}+D_{i j} \kappa_{j}^{0}+F_{i j} \kappa_{j}^{2} \quad(i, j=1,2,6), \\
& P_{i}=E_{i j} \epsilon_{j}^{0}+F_{i j} \kappa_{j}^{0}+H_{i j} \kappa_{j}^{2} \\
& Q_{2}=A_{4 j} \epsilon_{j}^{0}+D_{4 j} \kappa_{j}^{1}, \\
& Q_{1}=A_{5 j} \epsilon_{j}^{0}+D_{5 j} \kappa_{j}^{1}, \\
& K_{2}=D_{4 j} \epsilon_{j}^{0}+F_{4 j} \kappa_{j}^{1} \quad(j=4,5), \\
& K_{1}=D_{5 j} \epsilon_{j}^{0}+F_{5 j} \kappa_{j}^{1},
\end{aligned}
$$

where $\boldsymbol{A}_{i j}, \boldsymbol{B}_{i j}$, etc., are the laminate stiffnesses;

$$
\begin{aligned}
& \left(A_{i j}, B_{i j}, D_{i j}, E_{i j}, F_{i j}, H_{i j}\right) \\
& \quad=\sum_{k=1}^{N} \int_{\zeta k-1}^{\zeta k} Q_{i j}^{(k)}\left(1, \zeta, \zeta^{2}, \zeta^{3}, \zeta^{4}, \zeta^{6}\right) d \zeta, \\
& \quad \text { for } i, j=1,2,4,5,6 .
\end{aligned}
$$

\section{FIRST-ORDER SHEAR DEFORMATION (FSDT) AND CLASSICAL (CST) THEORIES}

For the sake of completeness and comparison, the equations of motion of the classical and first-order shell theories (see Ref. 1) are recorded here. 


\section{A. Classical theory}

$$
\begin{aligned}
& \frac{\partial N_{1}}{\partial x_{1}}+\frac{\partial N_{6}}{\partial x_{2}}+g_{1}=I_{1} \ddot{u}-I_{2} \frac{\partial \ddot{w}}{\partial x_{1}}, \\
& \frac{\partial N_{6}}{\partial x_{1}}+\frac{\partial N_{2}}{\partial x_{2}}+g_{2}=I_{1} \ddot{v}-I_{2} \frac{\partial \ddot{w}}{\partial x_{2}}, \\
& \frac{\partial^{2} M_{1}}{\partial x_{1}^{2}}+\frac{\partial^{2} M_{6}}{\partial x_{1} \partial x_{2}}+\frac{\partial^{2} M_{2}}{\partial x_{2}^{2}}-\frac{N_{1}}{R_{1}}-\frac{N_{2}}{R_{2}}+q \\
& \quad=I_{1} \ddot{w}+I_{2}\left(\frac{\partial \ddot{u}}{\partial x_{1}}+\frac{\partial \ddot{v}}{\partial x_{2}}\right)-I_{3}\left(\frac{\partial^{2} \ddot{w}}{\partial x_{1}^{2}}+\frac{\partial^{2} \ddot{w}}{\partial x_{2}^{2}}\right) .
\end{aligned}
$$

The resultants $N_{i}, M_{i}$ are given in Eqs. (6) and (8) with $E_{i j}=F_{i j}=0$, where the displacement functions $\phi_{1}$ and $\phi_{2}$ in this case are to be replaced by the expressions

$$
\phi_{i}=-\frac{\partial w}{\partial x_{i}} \quad(i=1,2)
$$

Note that in the present study the rotary inertia terms are included even in the classical theory.

\section{B. First-order theory}

$$
\begin{aligned}
& \frac{\partial N_{1}}{\partial x_{1}}+\frac{\partial N_{6}}{\partial x_{2}}+g_{1}=I_{1} \ddot{u}+I_{2} \ddot{\phi}_{1}, \\
& \frac{\partial N_{6}}{\partial x_{1}}+\frac{\partial N_{2}}{\partial x_{2}}+g_{2}=I_{1} \ddot{v}+I_{2} \ddot{\phi}_{2}, \\
& \frac{\partial Q_{1}}{\partial x_{1}}+\frac{\partial Q_{2}}{\partial x_{2}}-\frac{N_{1}}{R_{1}}-\frac{N_{2}}{R_{2}}+q=I_{1} \ddot{w}, \\
& \frac{\partial M_{1}}{\partial x_{1}}+\frac{\partial M_{6}}{\partial x_{2}}-Q_{1}+m_{1}=I_{2} \ddot{u}+I_{3} \ddot{\phi}_{1}, \\
& \frac{\partial M_{6}}{\partial x_{1}}+\frac{\partial M_{2}}{\partial x_{2}}-Q_{2}+m_{2}=I_{2} \ddot{v}+I_{3} \ddot{\phi}_{2} .
\end{aligned}
$$

The resultants $\left(N_{i}, M_{i}\right)$, can be expressed in terms of the strains in Eq. (3) by Eq. (8) with $E_{i j}=F_{i j}=0$. The resultant shear forces $Q_{1}$ and $Q_{2}$ are given by

$$
\begin{aligned}
& Q_{2}=K_{4}^{2} A_{44}\left(\phi_{2}+\frac{\partial w}{\partial x_{2}}-\frac{v}{R_{2}}\right), \\
& Q_{1}=K_{5}^{2} A_{55}\left(\phi_{1}+\frac{\partial w}{\partial x_{1}}-\frac{u}{R_{1}}\right),
\end{aligned}
$$

where $K_{4}^{2}, K_{5}^{2}$ are the shear correction factors.

Note that the equations of motion of the first-order theory can be deduced from those of the third-order theory by setting $K_{1}, K_{2}, P_{1}, P_{2}$, and $P_{6}$ to zero in Eq. (5) and $\kappa_{i}^{1}$ and $\kappa_{i}^{2}$ to zero in Eq. (3). Similarly, the classical theory can be obtained from the first-order theory by using Eq. (12).

\section{SOLUTION PROCEDURES}

Exact solutions for the dynamic response of the partial equations (5), (11), and (13) exist for simply supported (SS) shallow shell panels (see Refs. 11-13). The lamination scheme is of antisymmetric or symmetric cross-ply type where the following stiffnesses are zero:

$$
\begin{aligned}
& A_{i 6}=B_{i 6}=D_{i 6}=E_{i 6}=F_{i 6}=H_{i 6}=0 \quad(i=1,2), \\
& A_{45}=D_{45}=F_{45}=0 .
\end{aligned}
$$

The simply supported boundary conditions are assumed to be of the form:

$$
\begin{array}{cl}
\text { at } x_{1}=0, a & \\
v=w=N_{1}=M_{1}=\phi_{2}=P_{1}=0 & \text { HSDT, } \\
v=w=N_{1}=M_{1}=\phi_{2}=0 & \text { FSDT, } \\
v=w=N_{1}=M_{1}=0 & \text { CST; } \\
\text { at } x_{2}=0, b & \\
u=w=N_{2}=M_{2}=\phi_{1}=P_{2}=0 & \text { HSDT, } \\
u=w=N_{2}=M_{2}=\phi_{1}=0 & \text { FSDT, } \\
u=w=N_{2}=M_{2}=0 & \text { CST; }
\end{array}
$$

where $a$ and $b$ denote the lengths along the $x_{1}$ and $x_{2}$ directions, respectively.

\section{A. State-space approach}

Following the state space technique, ${ }^{14,15}$ we assume the following solution form that satisfies the boundary conditions in Eqs. (16) and (17):

$$
\begin{aligned}
& u\left(x_{1}, x_{2}, t\right)=\sum_{m, n=1}^{\infty} U_{m n}(t) f_{1}\left(x_{1}, x_{2}\right), \\
& v\left(x_{1}, x_{2}, t\right)=\sum_{m, n=1}^{\infty} V_{m n}(t) f_{2}\left(x_{1}, x_{2}\right), \\
& w\left(x_{1}, x_{2}, t\right)=\sum_{m, n=1}^{\infty} W_{m n}(t) f_{3}\left(x_{1}, x_{2}\right), \\
& \phi_{1}\left(x_{1}, x_{2}, t\right)=\sum_{m, n=1}^{\infty} X_{m n}(t) f_{1}\left(x_{1}, x_{2}\right), \\
& \phi_{2}\left(x_{1}, x_{2}, t\right)=\sum_{m, n=1}^{\infty} Y_{m n}(t) f_{2}\left(x_{1}, x_{2}\right),
\end{aligned}
$$

where

$$
\begin{aligned}
& f_{1}\left(x_{1}, x_{2}\right)=\cos \alpha x_{1} \sin \beta x_{2}, \\
& f_{2}\left(x_{1}, x_{2}\right)=\sin \alpha x_{1} \cos \beta x_{2}, \\
& f_{3}\left(x_{1}, x_{2}\right)=\sin \alpha x_{1} \sin \beta x_{2}, \quad \alpha=m \pi / a, \quad \beta=n \pi / b .
\end{aligned}
$$

The representation given for $u, v$, and $w$ in Eqs. (18) is also valid for CST.

Substituting Eqs. (18) into Eqs. (5), (11), and (13) results in the following set of equations

$$
[M]\left(\begin{array}{c}
\ddot{U}_{m n} \\
\ddot{V}_{m n} \\
\ddot{W}_{m n} \\
\ddot{X}_{m n} \\
\ddot{Y}_{m n}
\end{array}\right)+[C]\left(\begin{array}{c}
U_{m n} \\
V_{m n} \\
W_{m n} \\
X_{m n} \\
Y_{m n}
\end{array}\right)=\left(\begin{array}{c}
0 \\
0 \\
Q_{m n} \\
0 \\
0
\end{array}\right) \text { for any } m, n,
$$

for the HSDT and FSDT, and

$$
[M]\left(\begin{array}{c}
\ddot{U}_{m n} \\
\ddot{V}_{m n} \\
\ddot{W}_{m n}
\end{array}\right)+[C]\left(\begin{array}{c}
U_{m n} \\
V_{m n} \\
W_{m n}
\end{array}\right)=\left(\begin{array}{c}
0 \\
0 \\
Q_{m n}
\end{array}\right) \text { for any } m, n,
$$

for the CST.

Here, $Q_{m n}$ denotes the coefficient in the double Fourier expansion of the transverse load, 


$$
q\left(x_{1}, x_{2}, t\right)=\sum_{m, n=1}^{\infty} Q_{m n}(t) f_{3}\left(x_{1}, x_{2}\right),
$$

and $[C]$ and $[M]$ are the stiffness and mass matrices, respectively. The explicit form of $[C]$ and $[M]$ is different for HSDT and FSDT (see Ref. 8).

The linear systems of ordinary differential equations in Eqs. (19) and (20) can be solved using the state variables technique. According to this method we define the state vector $\mathbf{Z}(t)$ by

$$
\begin{aligned}
& Z_{1}=U_{m n}, \quad Z_{2}=\dot{U}_{m n}, \quad Z_{3}=V_{m n}, \quad Z_{4}=\dot{V}_{m n}, \\
& Z_{5}=W_{m n}, \quad Z_{6}=\dot{W}_{m n}, \quad Z_{7}=X_{m n}, \quad Z_{8}=\dot{X}_{m n}, \\
& Z_{9}=Y_{m n}, \quad Z_{10}=\dot{Y}_{m n},
\end{aligned}
$$

for the HSDT and FSDT, and

$$
\begin{array}{lll}
Z_{1}=U_{m n}, & Z_{2}=\dot{U}_{m n}, & Z_{3}=V_{m n}, \\
Z_{4}=\dot{V}_{m n}, & Z_{5}=W_{m n}, & Z_{6}=\dot{W}_{m n},
\end{array}
$$

for the CST.

Equations (19) and (20) can be written in the form:

$$
\dot{\mathbf{Z}}=A \mathbf{Z}+\mathbf{B} \text {, }
$$

where

$$
A=\left(\begin{array}{cccccccccc}
0 & 1 & 0 & 0 & 0 & 0 & 0 & 0 & 0 & 0 \\
L_{11} & 0 & L_{12} & 0 & L_{13} & 0 & L_{14} & 0 & L_{15} & 0 \\
0 & 0 & 0 & 1 & 0 & 0 & 0 & 0 & 0 & 0 \\
L_{21} & 0 & L_{22} & 0 & L_{23} & 0 & L_{24} & 0 & L_{25} & 0 \\
0 & 0 & 0 & 0 & 0 & 1 & 0 & 0 & 0 & 0 \\
L_{31} & 0 & L_{32} & 0 & L_{33} & 0 & L_{34} & 0 & L_{35} & 0 \\
0 & 0 & 0 & 0 & 0 & 0 & 0 & 1 & 0 & 0 \\
L_{41} & 0 & L_{42} & 0 & L_{43} & 0 & L_{44} & 0 & L_{45} & 0 \\
0 & 0 & 0 & 0 & 0 & 0 & 0 & 0 & 0 & 1 \\
L_{51} & 0 & L_{52} & 0 & L_{53} & 0 & L_{54} & 0 & L_{55} & 0
\end{array}\right),
$$

$\mathbf{B}=\left(0, b_{11}, 0, b_{21}, 0, b_{31}, 0, b_{41}, 0, b_{51}\right)^{T}$,

for the HSDT and FSDT, and

$$
\begin{aligned}
A & =\left(\begin{array}{cccccc}
0 & 1 & 0 & 0 & 0 & 0 \\
L_{11} & 0 & L_{12} & 0 & L_{13} & 0 \\
0 & 0 & 0 & 1 & 0 & 0 \\
L_{21} & 0 & L_{22} & 0 & L_{23} & 0 \\
0 & 0 & 0 & 0 & 0 & 1 \\
L_{31} & 0 & L_{32} & 0 & L_{33} & 0
\end{array}\right), \\
\mathbf{B} & =\left(0, b_{11}, 0, b_{21}, 0, b_{31}\right)^{T},
\end{aligned}
$$

where

$$
[L]=-[M]^{-1}[C]
$$

and

$$
\mathbf{b}=[M]^{-1}\left(\begin{array}{c}
0 \\
0 \\
Q_{m n} \\
0 \\
0
\end{array}\right),
$$

$$
\mathbf{b}=[M]^{-1}\left(\begin{array}{c}
0 \\
0 \\
Q_{m n}
\end{array}\right),
$$

for the CST. It should be noted that while the form of Eqs. (19), (25), (26), and (30) is the same for the FSDT and HSDT, the values of the variables in these equations are different for these two theories.

The solution to Eq. (24) is given by

$$
\mathbf{Z}(t)=e^{A\left(t-t_{0}\right)} \mathbf{Z}\left(t_{0}\right)+\int_{t_{0}}^{t} e^{A(t-\tau)} \mathbf{B}(\tau) d \tau,
$$

where $t_{0}$ is the initial time and $\mathbf{Z}\left(t_{0}\right)$ is the initial input vector. The operator $e^{A(t-\tau)}$ can be expressed in terms of the matrix of eigenvectors $[R]$ and distinct eigenvalues $\lambda_{i}$ associated with the matrix $A$ as

$$
e^{A(t-\tau)}=[R]\left(\begin{array}{ccc}
e^{\lambda_{1}(t-\tau)} & & 0 \\
& \ddots & \\
0 & & e^{\lambda_{n}(t-\tau)}
\end{array}\right)[R]^{-1},
$$

where $n=10$ for the HSDT and FSDT and $n=6$ for the CST.

\section{B. Separation of variables technique}

This method was used frequently in the literature to determine solutions to CST and FSDT. In this paper, only the HSDT will be treated. Assume a solution to Eq. (5) in the form

$$
\begin{aligned}
& u\left(x_{1}, x_{2}, t\right)=\sum_{m, n=1}^{\infty} U_{m n}\left(x_{1}, x_{2}\right) T_{m n}(t), \\
& v\left(x_{1}, x_{2}, t\right)=\sum_{m, n=1}^{\infty} V_{m n}\left(x_{1}, x_{2}\right) T_{m n}(t), \\
& w\left(x_{1}, x_{2}, t\right)=\sum_{m, n=1}^{\infty} W_{m n}\left(x_{1}, x_{2}\right) T_{m n}(t), \\
& \phi_{1}\left(x_{1}, x_{2}, t\right)=\sum_{m, n=1}^{\infty} \phi_{m n}^{1}\left(x_{1}, x_{2}\right) T_{m n}(t), \\
& \phi_{2}\left(x_{1}, x_{2}, t\right)=\sum_{m, n=1}^{\infty} \phi_{m n}^{2}\left(x_{1}, x_{2}\right) T_{m n}(t),
\end{aligned}
$$

where $\left(U_{m n}, V_{m n}, W_{m n}, \phi_{m n}^{1}, \phi_{m n}^{2}\right)$ are the principal modes of the laminated shell while $T_{m n}(t)$ is the unknown function of time $t$.

For the case of free vibration, the generalized coodinates $T_{m n}(t)$ and the principal modes associated with the homogeneous system of governing equations (5) and fulfilling the SS edge conditions defined in (16) and (17) may be expressed as

$$
\begin{array}{ll}
T_{m n}(t)=e^{i \omega_{m n} t}, & \\
U_{m n}=A_{m n} f_{1}\left(x_{1}, x_{2}\right), & V_{m n}=B_{m n} f_{2}\left(x_{1}, x_{2}\right), \\
W_{m n}=C_{m n} f_{3}\left(x_{1}, x_{2}\right) & \phi_{m n}^{1}=D_{m n} f_{1}\left(x_{1}, x_{2}\right), \\
\phi_{m n}^{2}=E_{m n} f_{2}\left(x_{1}, x_{2}\right), &
\end{array}
$$


where $\left(A_{m n}, B_{m n}, C_{m n}, D_{m n}, E_{m n}\right)$ are arbitrary constants. Substitution of Eqs. (34) and (35) into the equations of motion (5) yields a set of homogeneous equations that may be solved for the natural frequencies of vibration.

The orthogonality condition of the principal modes can be established with the result

$$
\begin{aligned}
\left(\omega_{m n}^{2}\right. & \left.-\omega_{r s}^{2}\right) \int_{0}^{a} \int_{0}^{b}\left\{\left(\bar{I}_{1} U_{m n}+\bar{I}_{2} \phi_{m n}^{1}-\bar{I}_{3} \frac{\partial W_{m n}}{\partial x_{1}}\right) U_{s r}+\left(\bar{I}_{1}^{\prime} V_{m n}+\bar{I}_{2}^{\prime} \phi_{m n}^{2}-\bar{I}_{3}^{\prime} \frac{\partial W_{m n}}{\partial x_{2}}\right) V_{s r}+\left[\bar{I}_{3} \frac{\partial U_{m n}}{\partial x_{1}}+\bar{I}_{5} \frac{\partial \phi_{m n}^{1}}{\partial x_{1}}\right.\right. \\
& \left.+\bar{I}_{3}^{\prime} \frac{\partial V_{m n}}{\partial x_{2}}+\bar{I}_{5}^{\prime} \frac{\partial \phi_{m n}^{2}}{\partial x_{2}}+I_{1} W_{m n}-\frac{16 I_{7}}{9 h^{4}}\left(\frac{\partial^{2} W_{m n}}{\partial x_{1}^{2}}+\frac{\partial^{2} W_{m n}}{\partial x_{2}^{2}}\right)\right] W_{s r}+\left(\bar{I}_{2} U_{m n}+\bar{I}_{4} \phi_{m n}^{1}-\bar{I}_{5} \frac{\partial W_{m n}}{\partial x_{1}}\right) \phi_{s r}^{1} \\
& \left.+\left(\bar{I}_{2}^{\prime} V_{m n}+\bar{I}_{4}^{\prime} \phi_{m n}^{2}-\bar{I}_{5}^{\prime} \frac{\partial W_{m n}}{\partial x_{2}}\right) \phi_{s r}^{2}\right\} d x_{1} d x_{2}=0
\end{aligned}
$$

The distributed loads are now expanded in a series of the principal modes:

$$
\begin{aligned}
& g_{1}=\sum_{m, n=1}^{\infty} f_{m n}(t)\left(\bar{I}_{1} U_{m n}+\bar{I}_{2} \phi_{m n}^{1}-\bar{I}_{3} \frac{\partial W_{m n}}{\partial x_{1}}\right), \\
& g_{2}=\sum_{m, n=1}^{\infty} f_{m n}(t)\left(\bar{I}_{1}^{\prime} V_{m n}+\bar{I}_{2}^{\prime} \phi_{m n}^{2}-\bar{I}_{3}^{\prime} \frac{\partial W_{m n}}{\partial x_{2}}\right), \\
& q=\sum_{m, n=1}^{\infty} f_{m n}(t)\left[\bar{I}_{3} \frac{\partial U_{m n}}{\partial x_{1}}+\bar{I}_{5} \frac{\partial \phi_{m n}^{1}}{\partial x_{1}}+\bar{I}_{3}^{\prime} \frac{\partial V_{m n}}{\partial x_{2}}+\bar{I}_{5}^{\prime} \frac{\partial \phi_{m n}^{2}}{\partial x_{2}}+I_{1} W_{m n}-\frac{16 I_{7}}{9 h^{4}}\left(\frac{\partial^{2} W_{m n}}{\partial x_{1}^{2}}+\frac{\partial^{2} W_{m n}}{\partial x_{2}^{2}}\right)\right] \\
& m_{1}=\sum_{m, n=1}^{\infty} f_{m n}(t)\left(\bar{I}_{2} U_{m n}+\bar{I}_{4} \phi_{m n}^{1}-\bar{I}_{5} \frac{\partial W_{m n}}{\partial x_{1}}\right), \\
& m_{2}=\sum_{m, n=1}^{\infty} f_{m n}(t)\left(\bar{I}_{2}^{\prime} V_{m n}+\bar{I}_{4}^{\prime} \phi_{m n}^{2}-\bar{I}_{5}^{\prime} \frac{\partial W_{m n}}{\partial x_{2}}\right) .
\end{aligned}
$$

The generalized forces $f_{m n}(t)$ are determined by making use of the orthogonality condition in Eq. (36). Multiplying Eq. (37a) by $U_{m n}$, Eq. (37b) by $V_{m n}$, Eq. (37c) by $W_{m n}$, Eq. (37d) by $\phi_{m n}^{1}$, and Eq. (37e) by $\phi_{m n}^{2}$, and adding the results, integrating over the plane area, and taking into account Eq. (36) leads to the result

$$
f_{m n}(t)=\frac{\int_{0}^{b} \int_{0}^{a}\left(g_{1} U_{m n}+g_{2} V_{m n}+q W_{m n}+m_{1} \phi_{m n}^{1}+m_{2} \phi_{m n}^{2}\right) d x_{1} d x_{2}}{N_{m n}}
$$

where

$$
\begin{aligned}
N_{m n}= & \int_{0}^{b} \int_{0}^{a}\left[\bar{I}_{1} U_{m n}^{2}+2 \bar{I}_{2} \phi_{m n}^{1} U_{m n}-\bar{I}_{3} \frac{\partial W_{m n}}{\partial x_{1}} U_{m n}+\bar{I}_{1}^{\prime} V_{m n}^{2}+2 \bar{I}_{2}^{\prime} \phi_{m n}^{2} V_{m n}-\bar{I}_{3}^{\prime} \frac{\partial W_{m n}}{\partial x_{2}} V_{m n}+\bar{I}_{3} \frac{\partial U_{m n}}{\partial x_{1}} W_{m n}\right. \\
& +\bar{I}_{5} \frac{\partial \phi_{m n}^{1}}{\partial x_{1}} W_{m n}+\bar{I}_{3}^{\prime} \frac{\partial V_{m n}}{\partial x_{2}} W_{m n}+\bar{I}_{5}^{\prime} \frac{\partial \phi_{m n}^{2}}{\partial x_{2}} W_{m n}+I_{1} W_{m n}^{2}-\frac{16 I_{7}}{9 h^{4}}\left(\frac{\partial^{2} W_{m n}}{\partial x_{1}^{2}}+\frac{\partial^{2} W_{m n}}{\partial x_{2}^{2}}\right) W_{m n} \\
& \left.+\bar{I}_{4}\left(\phi_{m n}^{1}\right)^{2}-\bar{I}_{5} \frac{\partial W}{\partial x_{1}} \phi_{m n}^{1}+\bar{I}_{4}^{\prime}\left(\phi_{m n}^{2}\right)^{2}-\bar{I}_{5} \frac{\partial W}{\partial x_{2}} \phi_{m n}^{2}\right] d x_{1} d x_{2} .
\end{aligned}
$$

Substituting Eqs. (34) into equations of motion (5), taking into account Eqs. (37), gives

$$
\ddot{T}_{m n}+\omega_{m n}^{2} T_{m n}=f_{m n} \text {, }
$$

for any $(m, n)$ and zero initial conditions. The solution to Eq. (40) is given by

$$
T_{m n}(t)=\frac{1}{\omega_{m n}} \int_{0}^{t} f_{m n}(\tau) \sin \omega_{m n}(t-\tau) d \tau
$$

For sinusoidal spatial distribution of load, $q(x, y, t)=q_{0} f_{3}\left(x_{1}, x_{2}\right) F(t),(m=n=1)$, the formal solution to the unknown functions may be expressed as

$$
\left(\begin{array}{c}
u \\
v \\
w \\
\phi_{1} \\
\phi_{2}
\end{array}\right)=\sum_{k=1}^{5} \frac{q_{0}}{J_{m n}(k) \omega_{m n}(k)}\left(\begin{array}{cc}
A_{m n}(k) & f_{1}\left(x_{1}, x_{2}\right) \\
B_{m n}(k) & f_{2}\left(x_{1}, x_{2}\right) \\
1 & f_{3}\left(x_{1}, x_{2}\right) \\
D_{m n}(k) & f_{1}\left(x_{1}, x_{2}\right) \\
E_{m n}(k) & f_{2}\left(x_{1}, x_{2}\right)
\end{array}\right) \int_{0}^{t} F(\tau) \sin \omega_{m n}(k)(t-\tau) d \tau
$$

where 


$$
\begin{aligned}
J_{m n}(k)= & \bar{I}_{1} A_{m n}^{2}(k)+2 \bar{I}_{2} A_{m n}(k) D_{m n}(k)-2 \bar{I}_{3} \alpha A_{m n}(k)+\bar{I}_{1}^{\prime} B_{m n}^{2}(k)+2 \bar{I}_{2}^{\prime} B_{m n}(k) E_{m n}(k) \\
& -2 \bar{I}_{3}^{\prime} \beta B_{m n}(k)-2 \bar{I}_{5} \alpha D_{m n}(k)-2 \bar{I}_{5}^{\prime} \beta E_{m n}(k)+I_{1}+\left(4 / 3 h^{2}\right)^{2} I_{7}\left(\alpha^{2}+\beta^{2}\right)+\bar{I}_{4} D_{m n}^{2}(k)+\bar{I}_{4}^{\prime} E_{m n}^{2}(k)
\end{aligned}
$$

Note that the solution in Eq. (42) is normalized with respect to $C_{m n}(k)$, the coefficients in the expansion of $W$.

\section{NUMERICAL RESULTS}

The two analytical solution procedures presented in the preceding sections yield the same solutions, although the space-state approach is more general. It is used to numerically evaluate the transient response of cross-ply shells. The following material properties of a lamina in its principal material coordinates are used:

$$
\begin{aligned}
& E_{1}=19.2 \times 10^{6} \mathrm{psi}, E_{2}=1.56 \times 10^{6} \mathrm{psi}, \\
& G_{12}=G_{13}=0.82 \times 10^{6} \mathrm{psi}, G_{23}=0.523 \times 10^{6} \mathrm{psi}, \\
& v_{12}=0.24 .
\end{aligned}
$$

The transverse deflection presented in the figures is evaluated at $(x, y, z)=(a / 2, b / 2, z)$. The stresses are nondimensionalized as follows:

$$
\begin{aligned}
& \bar{\sigma}_{1}=\frac{\sigma_{1}(a / 2, b / 2, h / 2)}{q_{0}}, \\
& \bar{\sigma}_{2}=\frac{\sigma_{2}(a / 2, b / 2, h / 2)}{q_{0}}, \\
& \bar{\sigma}_{4}=\frac{\sigma_{4}(a / 2,0,0)}{q_{0}} .
\end{aligned}
$$

In all calculations, unless stated otherwise, the following values are used (see Fig. 1):

$$
\begin{aligned}
& q_{0}=2000 \mathrm{psi}, \quad t_{1}=0.003 \mathrm{~s}, \\
& \rho=0.00012 \frac{\mathrm{lb}^{-\mathrm{s}^{2}}}{\mathrm{in}^{4}}, \quad a=b=20 \mathrm{in} ., \quad h=2 \mathrm{in} ., \\
& R_{1}=R_{2}=5 a \text { (spherical shells). }
\end{aligned}
$$

In all cases, sinusoidal distribution of loading in spatial domain and sine and triangular pulses in time domain are used.

$F(t)=\left\{\begin{array}{ll}\sin \left(\pi t / t_{1}\right), & 0 \leqslant t \leqslant t_{1} \\ 0, & t>t_{1}\end{array} \quad\right.$ (for sinusoidal loading),

$F(t)=\left\{\begin{array}{ll}1-t / t_{1}, & 0 \leqslant t \leqslant t_{1} \\ 0, & t>t_{1}\end{array} \quad\right.$ (for triangular loading).

For the first-order theory (FSDT), the shear correction coefficients are taken to be $K_{4}^{2}=K_{5}^{2}=5 / 6$.

The variation of center deflection with time for antisym-
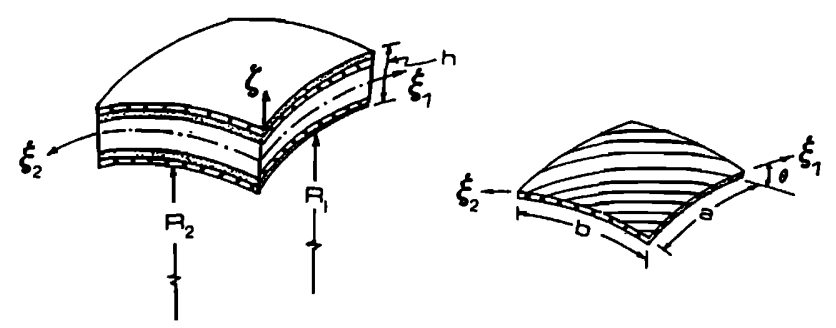

FIG. 1. Geometry and coordinate system of a double curved shell panel. metric cross-ply (0/90) and symmetric cross-ply (0/90/0) laminated spherical caps are shown in Figs. 2 and 3, respectively, for triangular and sinusoidal time variation of the sinusoidally distributed load. It is interesting to note that the response due to the triangular loading has larger amplitude than that due to the sinusoidal loading (both in time). Further, the amplitudes are smaller for symmetric cross-ply than for antisymmetric cross-ply laminates. It is also noted that the first-order and third-order theories predict almost the same response, while the classical theory differs both in amplitude and phase.

Similar results are presented for the normal stresses $\bar{\sigma}_{1}$
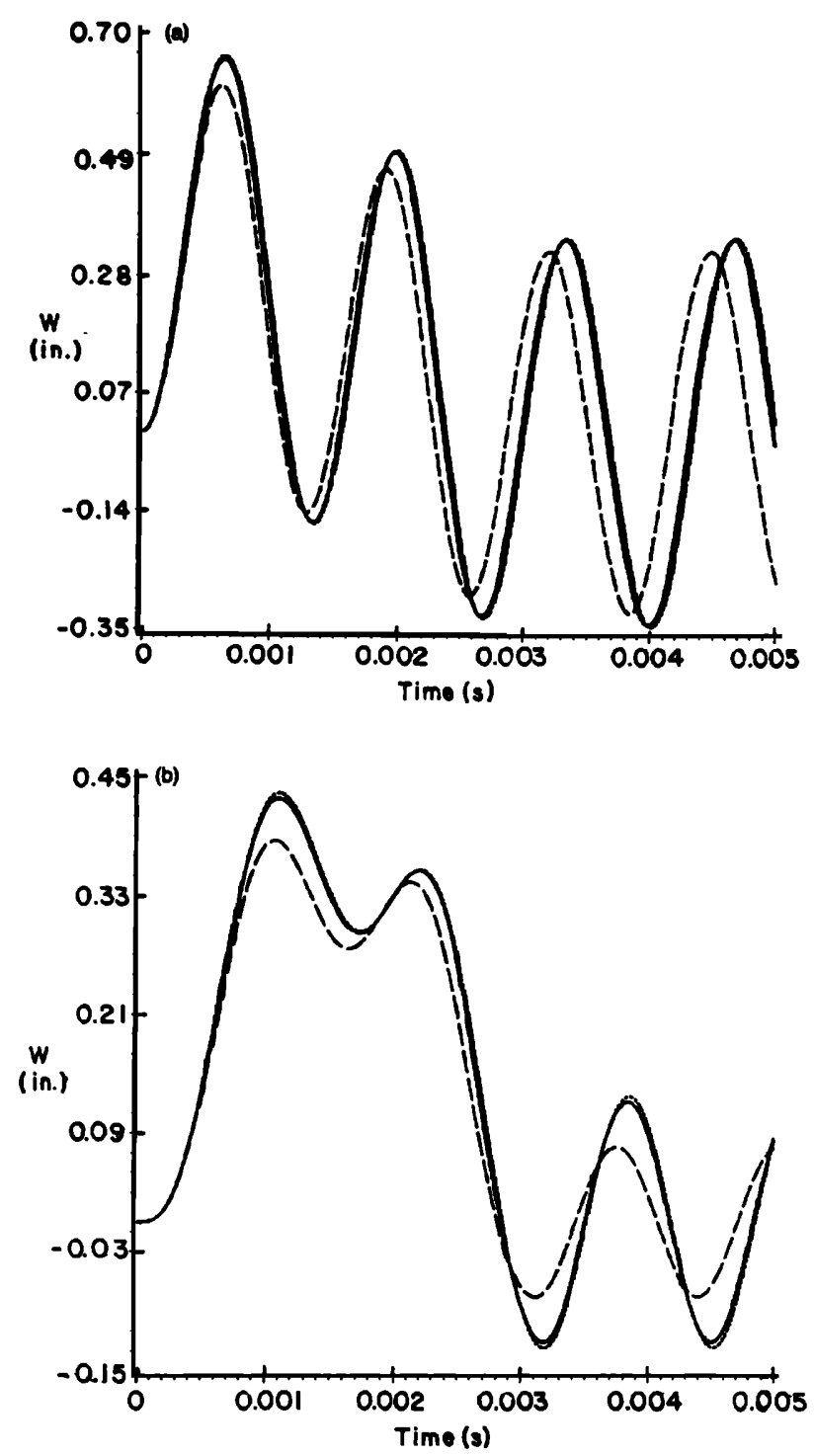

FIG. 2. Variation of the center deflection as a function of time, for twolayered $\left(0^{\circ} / 90^{\circ}\right)$ shells and two types of pulses (a) triangular (b) sine (-HSDT, - . FSDT, ---CST). 

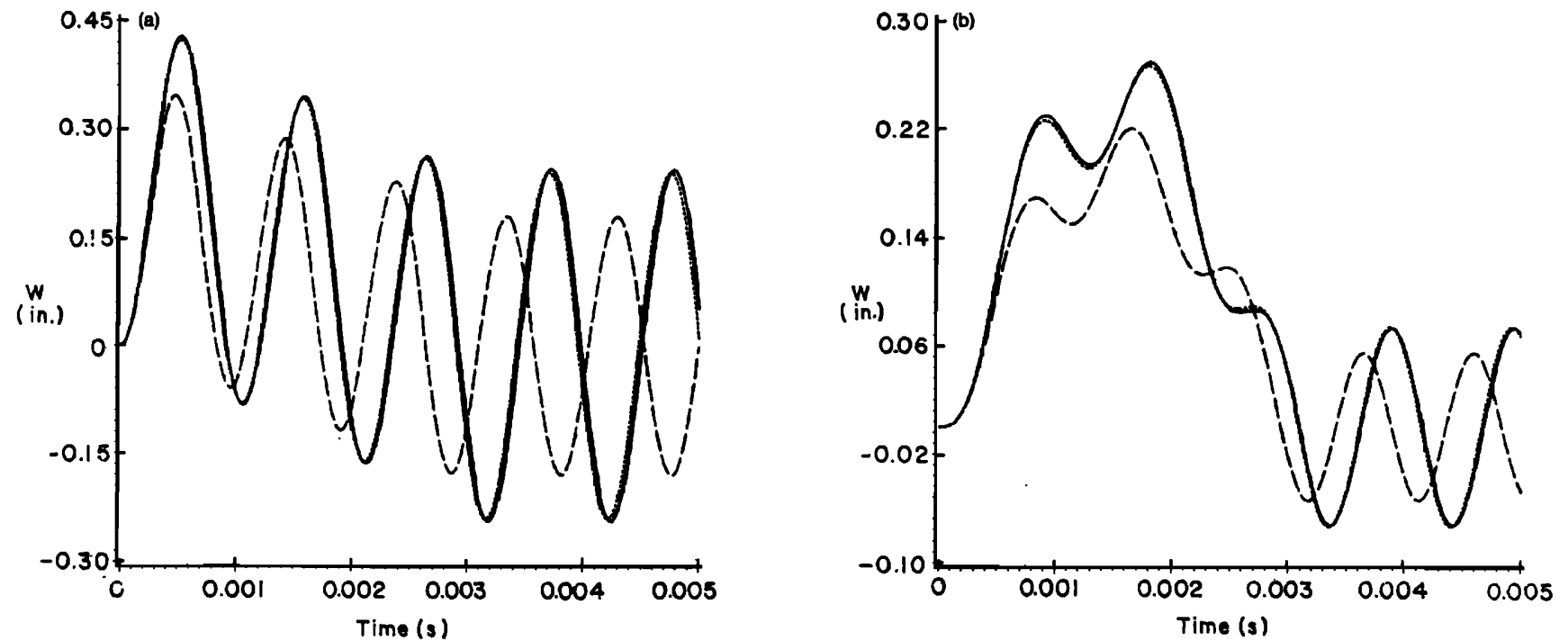

FIG. 3. Variation of the center deflection as a function of time, for three-layered $\left(0^{\circ} / 90^{\circ} / 0^{\circ}\right)$ shells and two types of pulses (a) triangular (b) sine (-HSDT, $\cdots$ FSDT, ---CST).
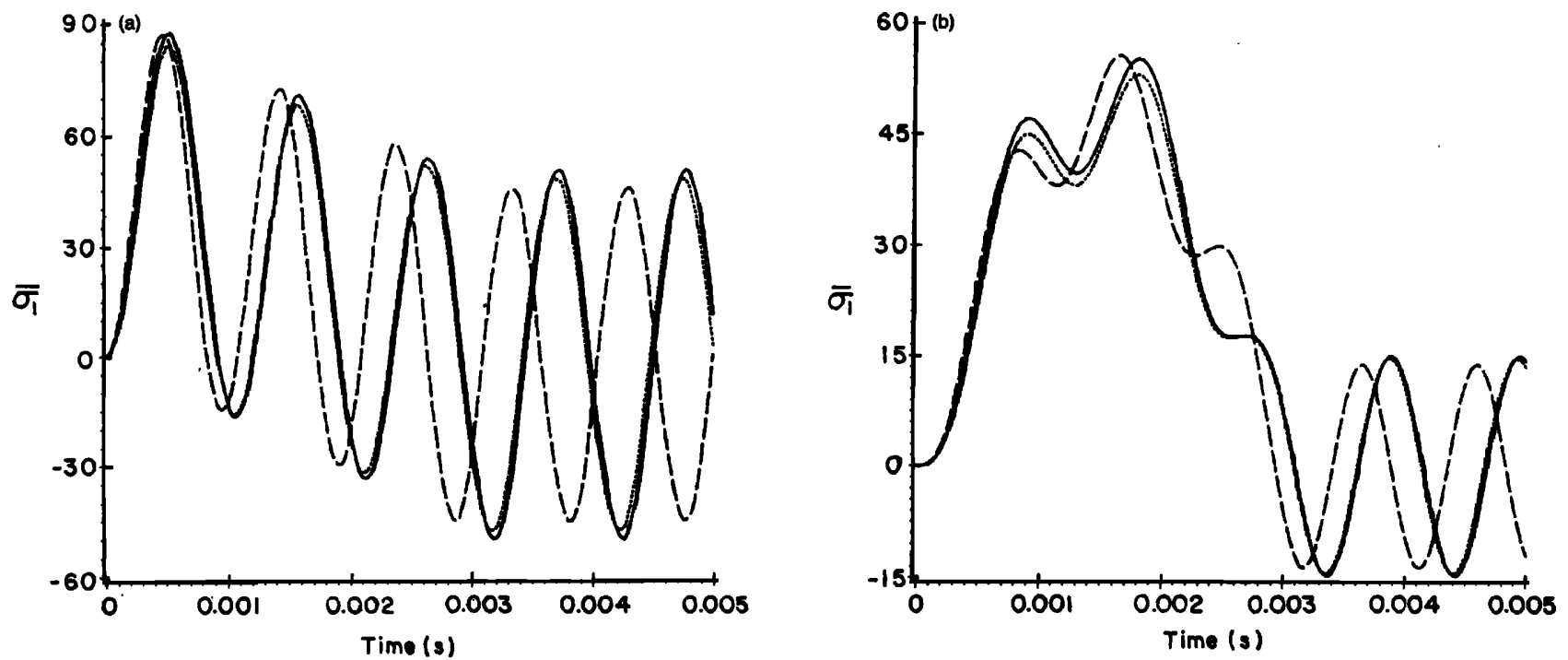

FIG. 4. Variation of the normal stress $\left(\bar{\sigma}_{1}\right)$ as a function of time, for three-layered $\left(0^{\circ} / 90^{\circ} / 0^{\circ}\right)$ shells and two types of pulses (a) triangular (b) sine (-HSDT, - - FSDT, ---CST).
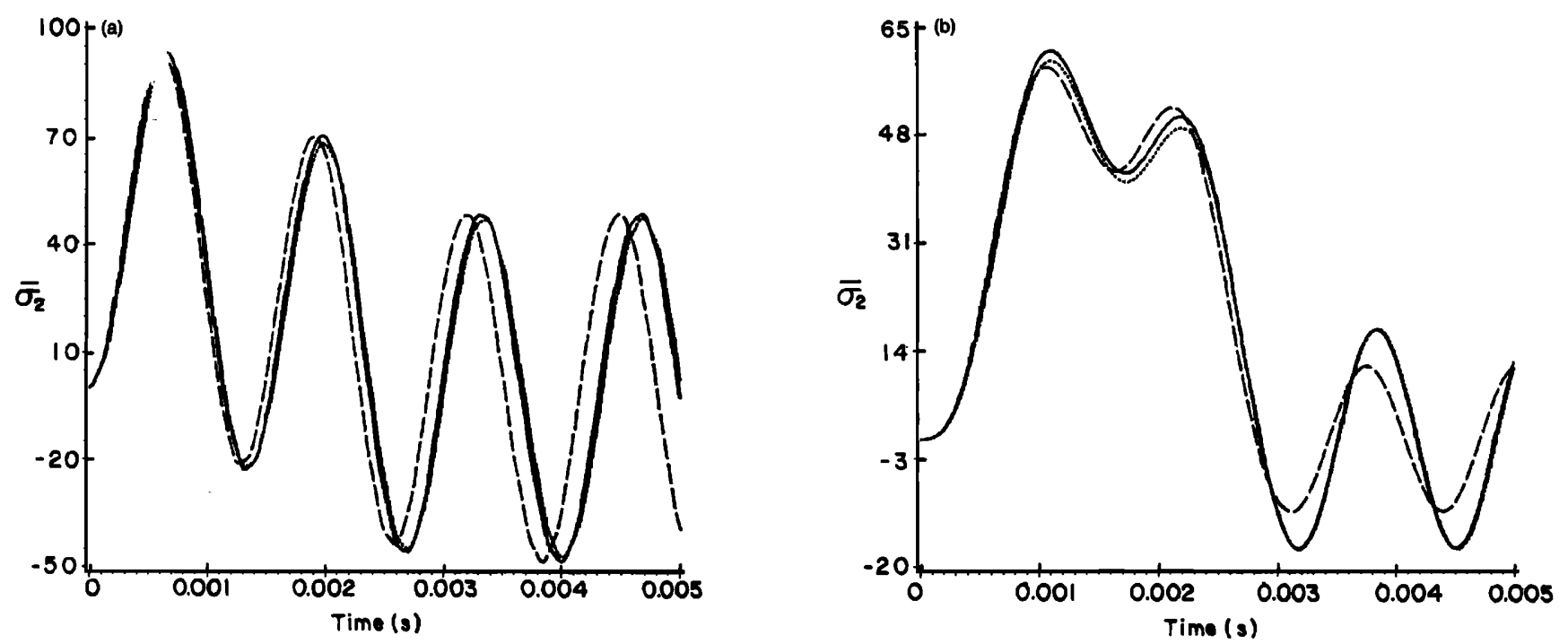

FIG. 5. Variation of the normal stress $\left(\bar{\sigma}_{2}\right)$ as a function of time for two-layered $\left(0^{\circ} / 90^{\circ}\right)$ shells and two types of pulses (a) triangular $(\mathrm{b})$ sine (-HSDT, $\cdots$ FSDT, ---CST). 

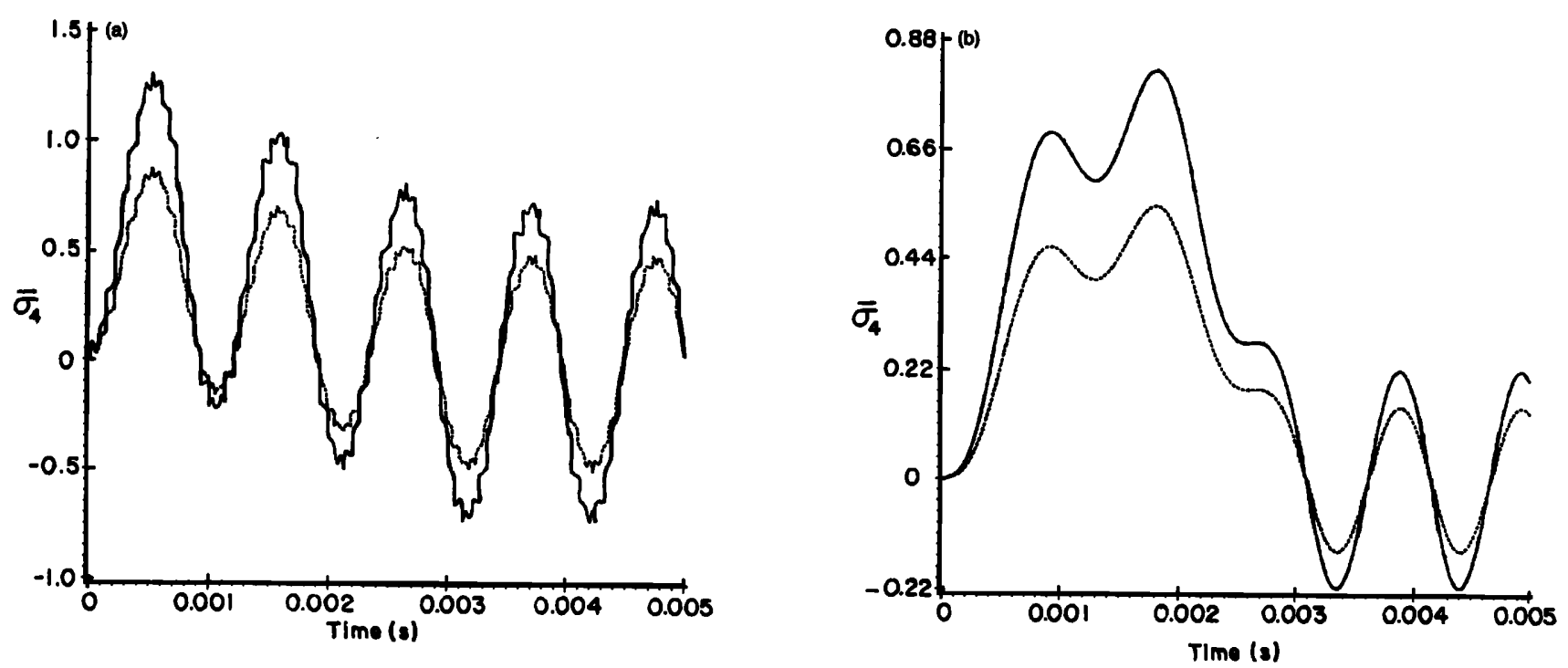

FIG. 6. Variation of the transverse shear stress $\left(\bar{\sigma}_{4}\right)$ as a function of time, for homogeneous shells and two types of pulses (a) triangular (b) sine (-HSDT, $\cdots$ FSDT).
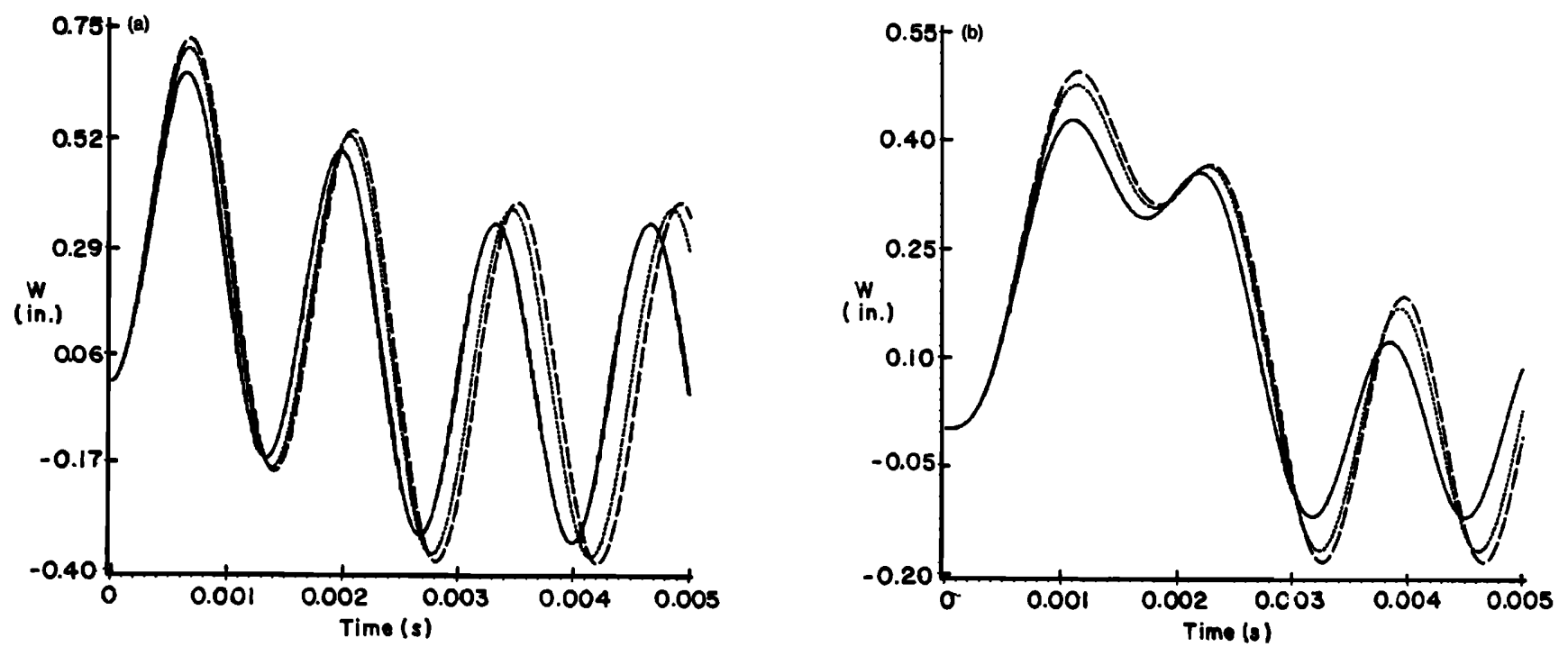

FIG. 7. Variation of the center deflection as a function of time, for two-layered $\left(0^{\circ} / 90^{\circ}\right)$ spherical shells for two types of pulses (a) triangular (b) sine $(-R=5 a, \cdots R=10 a, \cdots R=\infty)$.
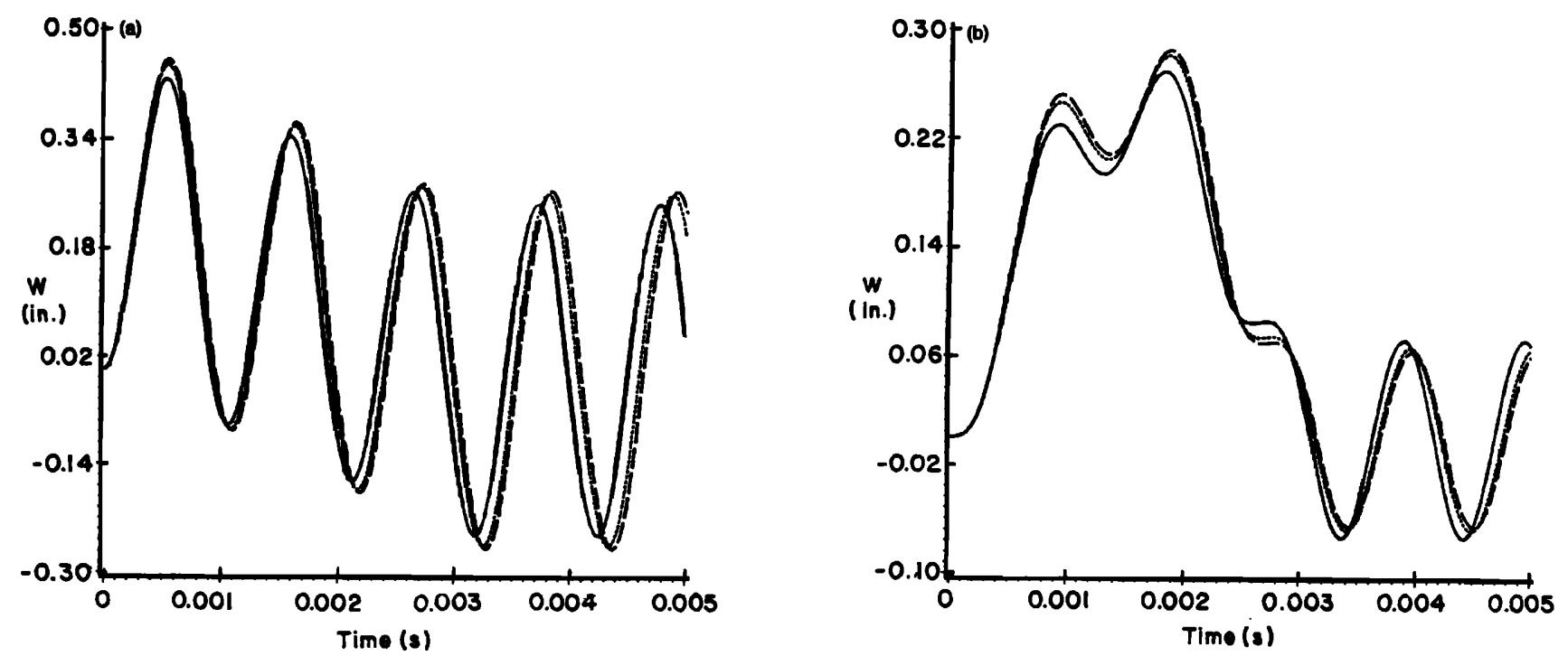

FIG. 8. Variation of the center deflection as a function of time, for three-layered $\left(0^{\circ} / 90^{\circ} / 0^{\circ}\right)$ spherical shells for two types of pulses (a) triangular (b) sine $(-R=5 a, \cdots R=10 a,--R=\infty)$. 
and $\bar{\sigma}_{2}$, and transverse shear stress $\bar{\sigma}_{4}$ in Figs. 4-6, respectively. Unlike the displacements, the stresses obtained using the three theories differ from each other, although the firstorder and third-order theories are relatively close for $\bar{\sigma}_{1}$ and $\bar{\sigma}_{2}$. The transverse shear stress predicted by the first-order theory differs significantly from that predicted by the thirdorder theory.

The effect of the shallowness of the shell on the center deflection of antisymmetric and symmetric cross-ply spherical caps is investigated, and the results are presented in Figs. 7 and 8 . All of the results are obtained using the higher-order theory. It is clear that the plate is relatively flexible when compared to the shell, as can be seen from the plots.

\section{v. CLOSURE}

The third-order shell theory of laminated composite shells is used to investigate the transient response of laminated spherical caps under a sinusoidally distributed transverse load that varies in time either as a triangular or sinusoidal load. Analytical solutions to simply supported laminated shells are developed using the state-space approach and separation of variables technique. The results are compared with those, also obtained in the present study, of the firstorder and classical shell theories. In general, the classical shell theory predicts deflections and stresses significantly different from those of the third-order theory. The thirdorder theory and first-order theory results are very close to each other. However, the third-order theory does not require the use of shear correction factors.
'J. N. Reddy, Energy and Variational Methods in Applied Mechanics (Wiley, New York, 1984).

${ }^{2}$ A. B. Basset, "On the Extension and Flexure of Cylindrical and Spherical Thin Elastic Shells," Philos. Trans. R. Soc. London, Ser. A 181 (6), 433480 (1890).

${ }^{4}$ F. B. Hildebrand, E. Reissner, and G. B. Thomas, "Notes on the Foundations of the Theory of Small Displacements of Orthotropic Shells," NACA Tech. Note No. 1833, March 1949.

${ }^{4} J$. L. Sanders, "Nonlinear Theories for Thin Shells," Q. Appl. Math. 21, 21-36 (1963).

${ }^{5}$ J. M. Whitney and C. T. Sun, "A Higher Order Theory for Extensional Motion of Laminated Anisotropic Shells and Plates," J. Sound Vib. 30, 85-97 (1973).

${ }^{6} \mathrm{~J}$. M. Whitney and C. T. Sun, "A Refined Theory for Laminated Anisotropic Cylindrical Shells,” J. Appl. Mech. 41, 47 (1974).

${ }^{7}$ J. N. Reddy, "A Simple Higher-Order Theory for Laminated Composite Plates," J. Appl. Mech. 51, 745-752 (1984).

${ }^{8}$ J. N. Reddy and C. F. Liu, "A Higher-Order Shear Deformation Theory of Laminated Elastic Shells," Int. J. Eng. Sci. 23 (3), 319-330 (1985).

${ }^{9} \mathrm{C}$. T. Sun and J. M. Whitney, "Forced Vibrations of Laminated Composite Plates in Cylindrical Bending," J. Acoust. Soc. Am. 55, 1003-1008 (1974)

${ }^{10} \mathrm{~J}$. M. Whitney and C. T. Sun, "Transient Response of Laminated Composite Plates Subjected to Transverse Dynamic Loading," J. Acoust. Soc. Am. 61, 101-104 (1977).

"A. L. Dobyns, "Analysis of Simply-Supported Orthotropic Plates Subjected to Static and Dynamic Loads," AIAA J. 19, 642-650 (1981).

'2J. N. Reddy, "Exact Solutions of Moderately Thick Laminated Shells," J. Eng. ASCE 110 (5), 794-809 (1984).

${ }^{13} \mathrm{~J}$. N. Reddy and C. F. Liu, A Higher-Order Theory for Geometrically Nonlinear Analysis of Composite Laminates (NASA Cr. Rept. 4656, March, 1987).

${ }^{14} \mathrm{~J}$. N. Franklin, Matrix Theory (Prentice-Hall, Englewood Cliffs, NJ, 1968).

${ }^{15}$ W. L. Brogan, Modern Control Theory (Prentice-Hall, Englewood Cliffs, NJ, 1985). 